\title{
GENERALIZED WEIGHT PROPERTIES OF THE RESULTANT OF $n+1$ POLYNOMIALS IN $n$ INDETERMINATES $\dagger$
}

\author{
BY \\ OSCAR ZARISKI
}

1. Introduction. The multiplicity of intersection of two plane algebraic curves, $f(x, y)=0$ and $g(x, y)=0$, at a common point $O(a, b), r$-fold for $f$ and $s$-fold for $g$, is not less than $r s$, and is greater than $r s$ if and only if the two curves have in common a principal tangent at $O$. The standard proof of this well known theorem of the theory of higher plane curves makes use of Puiseux expansions. If, namely, $R(x)=R(f, g)$ denotes the resultant of $f$ and $g$, considered as polynomials in $y$, and if $y_{1}, y_{2}, \cdots, y_{n}$ and $\bar{y}_{1}, \bar{y}_{2}, \cdots, \bar{y}_{m}$ are the roots of $f=0$ and $g=0$ respectively, then, the axes being in generic position, the intersection multiplicity at $O$ is defined as the multiplicity of the root $x=a$ of the resultant $R(x)$, and this multiplicity is found by substituting into the product $\prod_{i=1}^{n} \prod_{j=1}^{m}\left(y_{i}-\bar{y}_{j}\right)$ the Puiseux expansions of the roots $y_{i}$ and $\bar{y}_{j}$. A less known proof, in which the multiplicity to which the factor $x-a$ occurs in $R(x)$ is derived in a purely algebraic manner, was given by C. Segre. $\ddagger$ Following a procedure due to A. Voss, $\S$ Segre uses the Sylvester determinant and arrives at the required result by a skillful manipulation of the rows and columns.

In the first part of this paper $(\$ \S 2,3)$, we give a new proof of the property of the resultant $R(f, g)$ (see Theorem 1), which is implicitly contained in the quoted paper by $C$. Segre and of which the above intersection theorem is an immediate corollary. This proof makes use only of the intrinsic properties of the resultant and so contains the germ of an extension to the case of $n+1$ polynomials in $n$ variables. In the second part (\$\$4-9) we extend Theorem 1 to the resultant of $n+1$ polynomials (Theorem 6). From Theorem 6 follows as a corollary the analogous intersection theorem for hypersurfaces in $S_{n+1}$ $(\$ 9)$.

\section{Two Polynomials IN ONE VARIABLE}

2. A generalized weight property of the resultant. Let

$\dagger$ Presented to the Suciety, December 31, 1936; received by the editors June 18, 1936.

$\ddagger$ C. Segre, Le molteplicitd nelle intersezioni delle curve piane algebriche con alcune applicazioni ai principi della teoria di tali curve, Giornale de Matematiche di Battaglini, vol. 36 (1898).

$\S$ A. Voss, Über einen Fundamentalsatz aus der Theorie der algebraischen Functionen, Mathematische Annalen, vol. 27 (1886). 


$$
\begin{aligned}
& f=a_{0} y^{n}+a_{1} y^{n-1}+\cdots+a_{n}, \\
& g=b_{0} y^{m}+b_{1} y^{m-1}+\cdots+b_{m},
\end{aligned}
$$

be two polynomials, with literal coefficients, and let $R(f, g)$ be their resultant:

$$
R(f, g)=\sum a_{0}^{i_{0}} a_{1}^{i_{1}} \cdots a_{n}^{i_{n}} b_{0}^{j_{0}} b_{1}^{j_{1}} \cdots b_{m}^{i_{m}},
$$

where, by well known properties of $R$, we have

$$
\begin{aligned}
& i_{0}+i_{1}+\cdots+i_{n}=m, j_{0}+j_{1}+\cdots+j_{m}=n, \\
& i_{1}+2 i_{2}+\cdots+n i_{n}+j_{1}+2 j_{2}+\cdots+m j_{m}=m n .
\end{aligned}
$$

THEOREM 1. Let $r$ and $s$ be two non-negative integers, $r \leqq n, s \leqq m$. If we give to each coefficient $a_{i}\left(b_{j}\right)$ the weight $r-i(s-j)$ or zero, according as $r-i \geqq 0$ $(s-j \geqq 0)$ or $r-i \leqq 0(s-j \leqq 0)$, then the weight of any term in the resultant $R(f, g)$ is $\geqq r s$. The sum of terms of weight rs is given by the following expression:

where

$$
(-1)^{(m-s) r} R\left(f_{r}, g_{s}\right) R\left(f_{n-r}^{*}, g_{m-s}^{*}\right),
$$

$$
\begin{array}{ll}
f_{r}=a_{0} y^{r}+\cdots+a_{r}, & f_{n-r}^{*}=a_{r} y^{n-r}+\cdots+a_{n} ; \\
g_{s}=b_{0} y^{s}+\cdots+b_{s}, & g_{m-s}^{*}=b_{s} y^{m-s}+\cdots+b_{m} .
\end{array}
$$

We consider the polynomials

$$
\begin{aligned}
& \bar{f}=a_{0} t^{r} y^{n}+\cdots+a_{r-1} t y^{n-r+1}+a_{r} y^{n-r}+\cdots+a_{n}, \\
& \bar{g}=b_{0} t^{s} y^{m}+\cdots+b_{s-1} t y^{m-s+1}+b_{s} y^{m-s}+\cdots+b_{m},
\end{aligned}
$$

where $t$ is a new indeterminate. Let $t^{k}$ be the highest power of $t$ which divides the resultant $R(\bar{f}, \bar{g}), \bar{f}$ and $\bar{g}$ being considered as polynomials in $y$ :

$$
R(\bar{f}, \bar{g})=t^{k} R_{1}\left(a_{i}, b_{j}, t\right), . \quad R_{1}\left(a_{i}, b_{j}, 0\right) \neq 0 .
$$

By a well known property of the resultant, we have $R(\bar{f}, \bar{g})=A \bar{f}+B \bar{g}$, where $A$ and $B$ are polynomials in $y, a_{i}, b_{j}, t$, with integral coefficients; or using a familiar notation: $R(\bar{f}, \bar{g}) \equiv 0(\bar{f}, \bar{g})$. We put $\bar{f}=\bar{f}^{*}+a_{n}, \bar{g}=\bar{g}^{*}+b_{m}$. If we make the substitution $a_{n}=-\bar{f}^{*}, b_{m}=-\bar{g}^{*}$ in the identity $R(\bar{f}, \bar{g})=A \bar{f}+B \bar{g}$, then $\bar{f}$ and $\bar{g}$ vanish, and therefore also $t^{k} R_{1}\left(a_{i}, b_{j}, t\right)$ must vanish. Since $t$ is unaltered by the substitution, we have

$$
R_{1}\left(a_{0}, \cdots, a_{n-1},-\bar{f}^{*} ; b_{0}, \cdots, b_{m-1},-\bar{g}^{*} ; t\right)=0 .
$$

If we now order $R_{1}\left(a_{i}, b_{j}, t\right)$ according to the powers of $a_{n}+\bar{f}^{*}$ and $b_{m}+\bar{g}^{*}$, the constant term vanishes, and hence $R_{1}\left(a_{i}, b_{j}, t\right) \equiv 0(\bar{f}, \bar{g})$. $\uparrow$ Putting $t=0$,

$\dagger$ This proof that $t^{k} R_{1} \equiv 0(\bar{f}, \bar{g})$ implies $R_{1} \equiv 0(\bar{f}, \bar{g})$ is taken from van der Waerden, Moderne Algebra, II, p. 15 (quoted in the sequel as W.). Further on we shall use frequently the notion and properties of inertia forms as given in W., pp. 15-21. 
we find $R_{10}=R_{1}\left(a_{i}, b_{j}, 0\right) \equiv 0\left(f_{n-r}^{*}, g_{m-s}^{*}\right)$, and hence $R_{10}$ vanishes whenever $f_{n-r}^{*}$ and $g_{m-s}^{*}$ have a common factor of degree $\geqq 1$ in $y$. Consequently, by a well known property of the resultant, $R_{10}$ is divisible by $R\left(f_{n-r}^{*}, g_{m-s}^{*}\right)$, provided, however, that $n \neq r$ and $m \neq s$ (inequalities assuring the irreducibility of $\left.R\left(f_{n-r}^{*}, g_{m-s}^{*}\right)\right)$.

If we now consider the resultant $R(\bar{f}, \bar{g})$ of the following polynomials:

$$
\begin{aligned}
& \bar{f}=a_{0} y^{n}+\cdots+a_{r} y^{n-r}+t a_{r+1} y^{n-r-1}+\cdots+t^{n-r} a_{n}, \\
& \bar{g}=b_{0} y^{m}+\cdots+b_{s} y^{m-s}+t b_{s+1} y^{m-s-1}+\cdots+t^{m-s} b_{m},
\end{aligned}
$$

or, what is the same, the resultant of the polynomials

$$
\begin{gathered}
a_{n} t^{n-r} y^{n}+\cdots+a_{r+1} t y^{r+1}+a_{r} y^{r}+\cdots+a_{0}, \\
b_{m} t^{m-s} y^{m}+\cdots+b_{s+1} t y^{s+1}+b_{s} y^{s}+\cdots+b_{0},
\end{gathered}
$$

and if we put $R(\bar{f}, \bar{g})=t^{l} R_{2}\left(a_{i}, b_{j}, t\right)$ and $R_{20}=R_{2}\left(a_{i}, b_{j}, 0\right)$, where $t^{l}$ is the highest power of $t$ which divides $R(\bar{f}, \bar{g})$, we conclude as before that $R_{20}$ is divisible by the resultant of the polynomials

$$
\begin{aligned}
& a_{r} y^{r}+a_{r-1} y^{r-1}+\cdots+a_{0}, \\
& b_{s} y^{s}+b_{s-1} y^{s-1}+\cdots+b_{0} ;
\end{aligned}
$$

i.e., $R_{20}$ is divisible by $R\left(f_{r}, g_{s}\right)$, provided $r \neq 0$ and $s \neq 0$. But since

we have

$$
\bar{f}=t^{n-r} \bar{f}\left(\frac{y}{t}\right), \quad \bar{g}=t^{m-s} \bar{g}\left(\frac{y}{t}\right),
$$

$$
t^{r s} R(\bar{f}, \bar{g})=t^{(n-r)(m-s)} R(\bar{f}, \bar{g}),
$$

i.e., $R(\bar{f}, \bar{g})$ and $R(\bar{f}, \bar{g})$ differ only by a factor which is a power of $t$. Hence $R_{\mathbf{2 0}}=R_{10}$, and therefore $R_{10}$ is divisible by both $R\left(f_{n-r}^{*}, g_{m-s}^{*}\right)$ and $R\left(f_{r}, g_{s}\right)$.

Assuming that $r \neq 0, n, s \neq 0, m$, we have that $R\left(f_{n-r}^{*}, g_{m-s}^{*}\right)$ and $R\left(f_{r}, g_{s}\right)$ are irreducible and distinct polynomials in the coefficients $a_{i}, b_{j}$. [a $a_{0}$, for instance, actually occurs in $R\left(f_{r}, g_{s}\right)$, but does not occur in $R\left(f_{n-r}^{*}, g_{m-s}\right)$.] Hence $R_{10}$ is divisible by the product $R\left(f_{r}, g_{s}\right) \cdot R\left(f_{n-r}^{*}, g_{m-s}^{*}\right)$. Since $R(\bar{f}, \bar{g})$, and hence also $R_{10}$, is of degree $m$ in the coefficients of $f$ and of degree $n$ in the coefficients of $g$, we conclude that

$$
R_{10}=c R\left(f_{r}, g_{s}\right) R\left(f_{n-r}^{*}, g_{m-s}^{*}\right),
$$

where $c$ is a numerical factor (an integer).

Assume $r=0, s \neq m$. Then $f_{n-r}^{*}=f$ and $R\left(f_{n-r}^{*}, g_{m-s}^{*}\right)$ is irreducible and of degree $n$ in the coefficients of $g$, and consequently the quotient $R_{10} / R\left(f_{n-r}^{*}, g_{m-s}^{*}\right)$ is independent of the coefficients of $g$. On the other hand, $R(\bar{f}, \bar{g})$ contains 
the term $a_{0}{ }^{m} b_{m}{ }^{n}$, so that the exponent $k$ of $t$ in (1) equals 0 , and therefore $R_{10}$ can vanish only if $f$ and $g_{m-s}^{*}$ have a common zero or if $a_{0}=0$. It follows that also in this case $R_{10}=c a_{0}{ }^{8} R\left(f, g_{m-8}^{*}\right)=c \cdot R\left(f_{0}, g_{s}\right) R\left(f, g_{m-s}^{*}\right)$, where $c$ is an integer.

The case $s=0, r \neq n$ is treated in a similar manner.

$R_{10}$ is visibly given by the product $a_{0}{ }^{m} b_{m}{ }^{n}=R\left(f_{0}, g\right) R\left(f, g_{0}^{*}\right)$ if $r=0$, $s=m$, and a similar remark holds in the case $r=n, s=0$. Hence we have proved that in all cases

$$
R_{10}=c \cdot R\left(f_{r}, g_{s}\right) R\left(f_{n-r}^{*}, g_{m-s}^{*}\right),
$$

where $c$ is an integer.

The resultant $R(\bar{f}, \bar{g})$ can be obtained from $R(f, g)$ by replacing $a_{0}, a_{1}, \cdots$, $a_{r-1}$ and $b_{0}, b_{1}, \cdots, b_{s-1}$ by $a_{0} t^{r}, a_{1} t^{r-1}, \cdots, a_{r-1} t$ and $b_{0} t^{s}, b_{1} t^{s-1}, \cdots, b_{s-1} t$ respectively. Every term of $R(f, g)$ acquires then a factor $t^{w}$, where $w$ is the weight of this term as specified in the statement of Theorem 1. By (1), $R_{10}\left(=R_{1}\left(a_{i}, b_{j}, 0\right)\right)$ is the sum of all terms of $R(f, g)$ of lowest weight $k$, and since, always according to our definition of the weight, $R\left(f_{r}, g_{s}\right)$ is isobaric of weight $r s$, while $R\left(f_{-r}^{*}, g_{m-s}^{*}\right)$ is of weight zero, it follows that $k=r s$. This and the identity (2) complete the proof of our theorem.

To determine the numerical constant $c$, we take a special case, say $f=a_{0} y^{n}+a_{n}, g=b_{s} y^{m-s}$. Then $f_{r}=a_{0} y^{r}, g_{s}=b_{s}, f_{n-r}^{*}=a_{n}, g_{m-s}^{*}=b_{s} y^{m-s}$, and

$$
\begin{aligned}
R(f, g) & =(-1)^{(m-s) n} a_{0} a_{n}{ }^{m-s} b_{s}{ }^{n}, \\
R\left(f_{r}, g_{s}\right) & =a_{0}{ }^{8} b_{s}^{r}, R\left(f_{n-r}^{*}, g_{m-s}^{*}\right)=(-1)^{(n-r)(m-s)} a_{n}{ }^{m-s} b_{s}{ }^{n-r} .
\end{aligned}
$$

Hence, in this case we have

$$
R(f, g)=(-1)^{(m-s) r} R\left(f_{r}, g_{s}\right) R\left(f_{n-r}^{*}, g_{m-s}^{*}\right),
$$

and consequently $c=(-1)^{(m-s) r}$.

Remark. The resultant of the polynomials $f$ and $g$ coincides, to within the sign, with the resultant of the polynomials $a_{n} y^{n}+\cdots+a_{0}, b_{m} y^{m}+\cdots+b_{0}$. Applying our theorem to the last two polynomials, we see that it is permissible to interchange, in the statement of Theorem $1, a_{i}$ with $a_{n-i}$ and $b_{j}$ with $b_{n-j}$. This is equivalent to attaching the weights $r, r-1, \cdots, 1, s, s-1, \cdots, 1$ to $a_{n}, a_{n-1}, \cdots, a_{n-r+1}, b_{m}, b_{m-1}, \cdots, b_{m-s+1}$ respectively, and the weight 0 to the remaining coefficients.

3. The intersection multiplicity of two curves at a common point. The application of Theorem 1 toward the determination of the intersection multiplicity of two curves at a common point is immediate. If the coefficients $a_{i}$ and $b_{j}$ of the polynomials $f$ and $g$ are polynomials in $x$, and if the origin $O$ is a common point of the two curves $f=f(x, y)=0$, and $g=g(x, y)=0$, 
$r$-fold for $f$ and $s$-fold for $g$, then $a_{n}, a_{n-1}, \cdots, a_{n-r+1}$ are divisible by $x^{r}$, $x^{r-1}, \cdots, x$, respectively and $b_{m}, b_{m-1}, \cdots, b_{m-s+1}$ are divisible by $x^{s}, x^{s-1}, \cdots, x$, respectively. Hence every term of the resultant $R(f, g)=R(x)$ is divisible by $x^{w}$, where $w$ is the weight of the term as specified in the remark at the end of the preceding section. Since $w \geqq r s, x^{r s}$ divides $R(x)$. Let

$$
R(x)=\alpha x^{r s}+\text { terms of higher degree, }
$$

where $\alpha$ is a constant.

Let $f=\sum c_{i j} x^{i} y^{j}, g=\sum d_{i j} x^{i} y^{j}$. Then

$$
c_{i j}=\left[\frac{a_{n-j}(x)}{x^{r-j}}\right]_{x=0},
$$

for all $i$ and $j$ such that $i+j=r$; similarly

$$
d_{i j}=\left[\frac{b_{m-j}(x)}{x^{s-j}}\right]_{x=0},
$$

if $i+j=s$. Moreover, $c_{0, j}=a_{n-j}(0), j=r, r+1, \cdots, n$, and $d_{0, j}=b_{m-j}(0)$, $j=s, s+1, \cdots, m$. Applying Theorem 1, we find

$$
\alpha= \pm R\left(f_{r}, g_{\S}\right) R\left(f_{n-r}^{*}, g_{m-s}^{*}\right),
$$

where

and

$$
\begin{aligned}
& f_{r}=c_{r 0} x^{r}+c_{r-1,1} x^{r-1} y+\cdots+c_{0 r} y^{r}, \\
& g_{s}=d_{s 0} x^{s}+d_{s-1,1} x^{s-1} y+\cdots+d_{0 s} y^{s},
\end{aligned}
$$

$$
\begin{aligned}
& f_{n-r}^{*}=c_{0 r}+c_{0, r+1} y+\cdots+c_{0, n} y^{n-r}, \\
& g_{m-s}^{*}=d_{0 s}+d_{0, s+1} y+\cdots+d_{0, m} y^{m-s} .
\end{aligned}
$$

Here $R\left(f_{r}, g_{s}\right)=0$ if and only if the curves $f$ and $g$ have at the origin a common principal tangent. If $R\left(f_{r}, g_{s}\right) \neq 0$, then $R\left(f_{n-r}^{*}, g_{m-s}^{*}\right)=0$ if and only if the two curves have a common point on the $y$-axis outside the origin. Hence if the $y$-axis is generic and if there are no common principal tangents at $O$, then $\alpha \neq 0$ and the intersection multiplicity at $O$ equals $r s$.

\section{The general CASE of $n+1$ polynomials in $n$ INDETERminates}

4. Preliminary remarks on forms of inertia. Let $K$ be an underlying domain of integrity, and let $f_{1}, f_{2}, \cdots, f_{m}$ be polynomials in $x_{1}, x_{2}, \cdots, x_{n}$, with coefficients in a polynomial ring $K[t]=K\left[t_{1}, t_{2}, \cdots, t_{s}\right]$, where $t_{1}, \cdots, t_{s}$ are indeterminates. A polynomial $T$ in $K[t]$ is an inertia form of the polynomials $f_{1}, \cdots, f_{m}$, if it has the property:

$$
x_{i} \tau T \equiv 0\left(f_{1}, \cdots, f_{m}\right),
$$


for $i=1,2, \cdots, n$ and for some integer $\tau$, i.e., if $x_{i}{ }^{r} T$ belongs to the polynomial ideal generated by $f_{1}, \cdots, f_{m}$ in $K\left[t_{1}, \cdots, t_{s} ; x_{1}, \cdots, x_{n}\right]$. It follows from the definition that the inertia forms of $f_{1}, \cdots, f_{m}$ form an ideal $\mathfrak{T}$ in $K[t]$.

THEOREM 2. If for $\alpha=1,2, \cdots, n$ each polynomial $f_{j}$ is of the form: $f_{j}=t_{\alpha_{j}} x_{\alpha}^{\sigma j \alpha}+f_{j \alpha}^{*}, \sigma_{j \alpha} \geqq 0$, where $t_{\alpha_{1}}, \cdots, t_{\alpha_{m}}$ are distinct indeterminates in the set $t_{1}, \cdots, t_{s}$ and where $f_{j \alpha}{ }^{*}$ is a polynomial independent of $t_{\alpha_{1}}, \cdots, t_{\alpha_{m}}$, then $\mathfrak{T}$ is a prime ideal, and (3) holds for $i=1,2, \cdots, n$ if it holds for one value of $i$.

In order to prove $\nmid$ the theorem let, for instance, $f_{j}=t_{j} x_{1} \sigma_{i}+f_{i}^{*}$. If (3) holds for a given $i$ and for a given polynomial $T\left(t_{1}, t_{2}, \cdots, t_{m}, \cdots, t_{s}\right)$, then it follows by the substitution $t_{i}=-f_{i}^{*} / x_{1}^{\sigma_{i}}$ :

$$
T\left(-\frac{f_{1}^{*}}{x_{1}^{\sigma_{1}}},-\frac{f_{2}^{*}}{x_{1}^{\sigma_{2}}}, \cdots,-\frac{f_{m}^{*}}{x_{1}^{\sigma_{m}}}, \cdots, t_{t}\right)=0 .
$$

Conversely, if a polynomial $T\left(t_{1}, \cdots, t_{s}\right)$ vanishes identically after the substitution $t_{j}=-f_{j}^{*} / x_{1}{ }^{\sigma_{i}}$, then $T$ satisfies (3) for $i=1$. Under the assumption made in the above theorem, it follows immediately that (4) is a necessary and sufficient condition in order that $T$ be a form of inertia. Hence $\mathfrak{T}$ is a prime ideal and $T$ is a form of inertia if (3) holds for $i=1$.

COROLlaRy. If $\sigma_{1}=\sigma_{2}=\cdots=\sigma_{m}=0$, then any form of inertia $T$ satisfies (3) with $\tau=0$.

If the polynomials $f_{1}, f_{2}, \cdots, f_{m}$ are homogeneous in $x_{1}, \cdots, x_{n}$, then it is well known that the vanishing of all the inertia forms for special values $t_{j}{ }^{0}$ of the parameters $t_{j}$ is a necessary and sufficient condition that the equations $f_{1}\left(x_{i} ; t_{i}^{0}\right)=0, f_{2}\left(x_{i} ; t_{i}^{0}\right)=0, \cdots, f_{m}\left(x_{i} ; t_{i}^{0}\right)=0$ have a non-trivial solution (not all $x_{i}=0$ ) (see W., p. 16).

For non-homogeneous polynomials the following theorem holds:

THEOREM 3.1. Let $f_{i}$ contain terms of lowest degree $s_{i}$ in $x_{1}, \cdots, x_{n}$ :

$$
f_{i}=f_{i, s i}\left(x_{1}, \cdots, x_{n}\right)+f_{i, s i+1}\left(x_{1}, \cdots, x_{n}\right)+\cdots,
$$

where $f_{i, k}$ is homogeneous of degree $k$ in $x_{1}, \cdots, x_{n}$, and let us consider the homogeneous polynomials:

$$
\bar{f}_{i}=x_{0}^{l_{i-s i}} f_{i, s i}\left(x_{1}, \cdots, x_{n}\right)+x_{0}^{l_{i-s i-1}} f_{i, s i+1}\left(x_{1}, \cdots, x_{n}\right)+\cdots,
$$

where $x_{0}$ is an indeterminate and $l_{i}$ is the degree of $f_{i}$. The vanishing of all the inertia forms of $f_{1}, f_{2}, \cdots, f_{m}$ for special values of the parameters $t_{j}$ is a sufficient condition in order that (a) either the equations $\bar{f}_{1}=0, \cdots, \bar{f}_{m}=0$ have a non-

† Compare W., p. 15. 
trivial solution different from $x_{0}=1, x_{1}=\cdots=x_{n}=0$; or that $(\mathrm{b})$ the equations $f_{1, s_{1}}\left(x_{1}, \cdots, x_{n}\right)=0, \cdots, f_{m, s_{m}}\left(x_{1}, \cdots, x_{n}\right)=0$ have a non-trivial solution (in a suitable extension field of $K$ ).

The converse holds only under certain restrictions:

THEOREM 3.2. If (a) holds and if the coefficients of $x_{1}^{l_{i}}, \cdots, x_{n}{ }^{l_{i}}$ in $f_{i}$ $(i=1,2, \cdots, m)$ are indeterminates which do not occur in other terms of $f_{i}$, then the inertia forms of $f_{1}, \cdots, f_{m}$ all vanish.

THEOREM 3.3. If (b) holds, and if the coefficients of $x_{1^{8 i}}, \cdots, x_{n}^{s^{s i}}$ in $f_{i}$ $(i=1,2, \cdots, m)$ are indeterminates which do not occur in other terms of $f_{i}$, then the inertia forms of $f_{1}, \cdots, f_{m}$ all vanish.

$\bar{f}_{1}, \cdots, \bar{f}_{m}$, considered as polynomials in $x_{0}$, possess a resultant system

$$
\phi_{1}\left(x_{1}, \cdots, x_{n}\right), \cdots, \phi_{h}\left(x_{1}, \cdots, x_{n}\right) \text {, }
$$

where the $\phi_{i}$ 's are homogeneous polynomials. Since $\phi_{i} \equiv 0\left(\bar{f}_{1}, \cdots, \bar{f}_{m}\right)$, we have for every inertia form $T$ of the polynomials $\phi_{i}: x_{i}{ }^{\sigma} T \equiv 0\left(\bar{f}_{1}, \cdots, \bar{f}_{m}\right)$, $j=1,2, \cdots, n$. Putting $x_{0}=1$, we see that $T$ is also an inertia form of the polynomials $f_{1}, \cdots, f_{m}$.

Let all the inertia forms of $f_{1}, \cdots, f_{m}$ vanish for special values of the parameters $t_{j}$. Then for these special values of the $t_{j}$ 's also the inertia forms of $\phi_{1}, \cdots, \phi_{h}$ all vanish, the homogeneous equations $\phi_{1}=0, \cdots, \phi_{h}=0$ have a non-trivial solution, and consequently, by known properties of the resultant system $\phi_{1}, \cdots, \phi_{h}$, the alternatives (a) and (b) of Theorem 3.1 follow.

If $T$ is an inertia form of $f_{1}, \cdots, f_{m}$, then passing to the homogeneous polynomials $\bar{f}_{1}, \cdots, \bar{f}_{m}$, it is found that $\left(x_{0} x_{i}\right)^{\sigma} T \equiv 0\left(\bar{f}_{1}, \cdots, \bar{f}_{m}\right)$, for $i=1,2, \cdots, n$ and for some $\sigma$. Under the hypothesis of Theorem 3.2 concerning the coefficients of $x_{1}^{l_{i}}, \cdots, x_{n}{ }^{l_{i}}$, we can repeat the reasoning of the proof of Theorem 2, and it follows that $x_{i} \rho T \equiv 0\left(\bar{f}_{1}, \cdots, \bar{f}_{m}\right)$, for $i=1,2, \cdots, n$ and for some $\rho$. Hence if (a) holds, then $T=0$.

For the proof of Theorem 3.3, let $x_{1}^{0}, \cdots, x_{n}^{0}$ be a non-trivial solution of the equations $f_{1, s_{1}}=0, \cdots, f_{m, s_{m}}=0$, and let, for instance, $x_{1}^{0} \neq 0$. We make the following change of indeterminates:

Then

$$
x_{1}=y_{1}, x_{2}=y_{2} y_{1}, \cdots, x_{n}=y_{n} y_{1} .
$$

$$
\begin{aligned}
f_{i} & =y_{1}^{s i} f_{1,8 i}\left(1, y_{2}, \cdots, y_{n}\right)+y_{1}^{s i+1} f_{1,8,+1}\left(1, y_{2}, \cdots, y_{n}\right)+\cdots \\
& =y_{1}^{s i} \psi_{i}\left(y_{1}, \cdots, y_{n}\right),
\end{aligned}
$$

and if $T$ is an inertia form of $f_{1}(x), \cdots, f_{m}(x)$, then $y_{1}{ }^{\sigma} T \equiv 0\left(\psi_{1}, \cdots, \psi_{m}\right)$. Under the hypothesis of Theorem 3.3, the constant terms in $\psi_{1}, \psi_{2}, \cdots, \psi_{m}$ are in- 
determinates, and hence, by the corollary to Theorem $2, T \equiv 0\left(\psi_{1}, \cdots, \psi_{m}\right)$. Since for $t_{i}=t_{i}{ }^{0}$, the equations $\psi_{1}=0, \cdots, \psi_{m}=0$ have the solution $y_{1}{ }^{0}=0$, $y_{2}^{0}=x_{2}^{0} / x_{1}^{0}, \cdots, y_{n}=x_{n}^{0} / x_{1}^{0}$, it follows that $T\left(t_{1}^{0}, \cdots, t_{s}^{0}\right)=0$.

5 . The inertia forms of some special set of $n+1$ polynomials in $n$ indeterminates. The theorems of the preceding section are applicable in the special case when $m=n+1$ and when each $f_{i}$ is a polynomial with literal coefficients in which all the terms of degree $\left\langle s_{i} \leqq l_{i}\right.$ are missing, $l_{i}$ being the degree of $f_{i}$ :

$$
f_{i}=\sum_{(j)} a_{j_{1} j_{2} \cdots j_{n}}^{(i)} x_{1}^{j_{1}} x_{2}^{j_{2}} \cdots x_{n}^{j_{n}}, \quad s_{i} \leqq j_{1}+\cdots+j_{n} \leqq l_{i} .
$$

If $s_{1}=s_{2}=\cdots=s_{n+1}=0$, then the ideal of the inertia forms is a principal ideal $(R)$, where $R$ is the resultant of $f_{1}, f_{2}, \cdots, f_{n+1} . R$ is an irreducible polynomial homogeneous of degree $l_{2} \cdots l_{n+1}$ in the coefficients of $f_{1}$, homogeneous of degree $l_{1} l_{3} \cdots l_{n+1}$ in the coefficients of $f_{2}$, etc. Finally, by the corollary to Theorem 2, $R \equiv 0\left(f_{1}, f_{2}, \cdots, f_{n+1}\right)$, and the vanishing of $R$ for special values of the coefficients $a_{(j)}^{(i)}$ is a necessary and sufficient condition in order that the polynomials $f_{1}, f_{2}, \cdots, f_{n+1}$, rendered homogeneous, have a common non-trivial zero (see W., p. 20).

We prove the following theorems in the case when $s_{1}, s_{2}, \cdots, s_{n+1}$ are not necessarily all zero:

THEOREM 4. Let $e_{i}\left(=a_{l_{i}}^{(i)} \ldots 0\right)$ be the coefficient of $x_{1}{ }^{l_{i}}$ in $f_{i}$. If $s_{n+1}<l_{n+1}$, then any inertia form of $f_{1}, f_{2}, \cdots, f_{n+1}$ which does not vanish identically, must be of degree $>0$ in each of the coefficients $e_{1}, e_{2}, \cdots, e_{n}$.

COROLLARY. If one at least of the polynomials $f_{1}, \cdots, f_{n+1}$ is non-homogeneous, the ideal $\mathfrak{T}$ of their inertia forms is a principal ideal. $\dagger$

The proof is similar to the one given in W., pp. 16-17, in the case $s_{1}=\cdots=s_{n+1}=0$, only with a slightly different specialization of the coefficients $a_{(j)}^{(i)}$. Assume that there exists an inertia form $T$, not identically zero, which is independent of $e_{1}$. Putting $f_{i}=e_{i} x_{1}{ }^{l_{i}}+f_{i}^{*}$, and applying (4) (where $\sigma_{i}$ should be replaced by $l_{i}$ ), we see that $T$ cannot be independent of all the coefficients $e_{2}, \cdots, e_{n+1}$ (since $T$ is not identically zero) and we conclude that the quotients

$$
f_{2}^{*} / x_{1}^{l_{2}}, \cdots, f_{n+1}^{*} / x_{1}^{l_{n+1}}
$$

are algebraically dependent in $K\left[a_{(j)}^{(i)}\right], K$ being the ring of natural integers. By a lemma proved in W., p. 17, these quotients remain algebraically depend-

$\dagger$ If all the polynomials $f_{i}$ are homogeneous, then $\mathfrak{T}$ contains the resultant of any $n$ of these polynomials and is therefore not a principal ideal. 
ent after an arbitrary specialization $a_{(j)}^{(i)}=\alpha_{(j)}^{(i)}\left(\alpha_{(j)}^{(i)}<K\right)$. Let us take for $f_{1}, f_{2}, \cdots, f_{n+1}$ the special set of polynomials $x_{1}^{l_{1}}, x_{1}{ }^{l_{2}-1} x_{2}, \cdots, x_{1}{ }^{l_{n}-1} x_{n}$, $x_{1}^{l n+1-1}$, observing that the specialization $f_{n+1}=x_{1}{ }^{l_{n+1}-1}$ is permissible, since, by hypothesis, $f_{n+1}$ is not homogeneous. The above quotients become

$$
x_{2} / x_{1}, \cdots, x_{n} / x_{1}, 1 / x_{1},
$$

and since these are evidently algebraically independent, our assumption that $T$ is independent of $e_{1}$ leads to a contradiction.

The corollary now follows in exactly the same manner as in W., p. 19.

Let $(D)$ be the principal ideal of the inertia forms of the polynomials $f_{1}, f_{2}, \cdots, f_{n+1} . D$, if it is not identically zero, is an irreducible polynomial in the coefficients $a_{(j)}^{(i)}$. We next prove that indeed $D$ is not identically zero, i.e., that there exist inertia forms of $f_{1}, \cdots, f_{n+1}$ which are not identically zero.

If $\phi_{1}, \cdots, \phi_{n+1}$ denote general polynomials in $x_{1}, \cdots, x_{n}$ with literal coefficients, of degree $l_{1}, l_{2}, \cdots, l_{n+1}$ respectively, we can write $\phi_{i}=\psi_{i}+f_{i}$, where $f_{1}, \cdots, f_{n+1}$ are our given polynomials and where $\psi_{i}$ is of degree $s_{i}-1$. Let $\phi_{i}=\sum a_{j_{1}}^{(i)} \ldots j_{n} x_{1}{ }_{1}{ }_{1} \cdots x_{n}{ }^{j_{n}}, 0 \leqq j_{1}+\cdots+j_{n} \leqq l_{i}$. Let $t$ be a parameter, and let $\phi_{i}{ }^{t}$ be the polynomial obtained from $\phi_{i}$ by replacing each coefficient $a_{j_{1}}^{(i)} \ldots j_{n}$ by $t^{t_{i}-j_{1}-\cdots-j_{n}} a_{j_{1}}^{(i)} \cdots j_{n}$, if $s_{i}>j_{1}+\cdots+j_{n}$, i.e., if $a_{j_{1}}^{(i)} \cdots j_{n}$ is the coefficient of a term of the polynomial $\psi_{i}$, while the coefficients of $f_{i}$ remain unaltered. Let $R_{t}=R\left(\phi_{1}{ }^{t}, \cdots, \phi_{n+1}^{t}\right)$ be the resultant of the $\phi_{i}{ }^{t}$ 's considered as polynomials in $x_{1}, \cdots, x_{n}$, and let $t^{\alpha}, \alpha \geqq 0$, be the highest power of $t$ which divides $R_{t}$ :

$$
R_{t}=t^{\alpha} R^{(1)}\left(t, a_{(j)}^{(i)}\right)=t^{\alpha} R_{t}^{(1)} .
$$

Since each polynomial $\phi_{i}{ }^{t}$ contains the terms $x_{1}{ }^{l_{i}}, \cdots, x_{n}{ }^{l_{i}}$, whose coefficients are indeterminates, it follows by Theorem 2, that the ideal of the inertia forms of $\phi_{1}{ }^{t}, \cdots, \phi_{n+1}^{t}$ is prime. Now, no power of $t$ is an inertia form of $\phi_{1}{ }^{t}, \cdots, \phi_{n+1}^{t}$, because otherwise, for $t=1$, it would follow that 1 is an inertia form of $\phi_{1}, \cdots, \phi_{n+1}$, and this is impossible. Hence, since $t^{\alpha} R_{t}^{(1)}$ is an inertia form of $\phi_{1}{ }^{t}, \cdots, \phi_{n+1}^{t}$, it follows that also $R_{t}^{(1)}$ is an inertia form. For $t=0$, we have $\phi_{i}{ }^{0}=f_{i}$, and $R_{0}{ }^{(1)}$ is therefore an inertia form of $f_{1}, \cdots, f_{n+1}$ which does not vanish identically.

6. The resultant $R\left(\phi_{1}, \cdots, \phi_{n+1}\right)$ as an isobaric function of the coeffcients $a_{(j)}^{(i)}$. Let $\phi_{1}, \cdots, \phi_{n+1}$ denote, as in the preceding section, general polynomials in the $n$ variables $x_{1}, \cdots, x_{n}$, of degree $l_{1}, \cdots, l_{n+1}$ respectively, and let $R\left(\phi_{1}, \cdots, \phi_{n+1}\right)=R\left(a_{(j)}^{(i)}\right)$ be their resultant. It is clear that $R\left(\cdots, t^{j_{1}+\cdots+j_{n}} a_{j_{1}}^{(i)} \cdots j_{n}, \cdots\right)$ is the resultant of $\phi_{1}\left(x_{1} t, \cdots, x_{n+1} t\right), \cdots$, $\phi_{n+1}\left(x_{1} t, \cdots, x_{n+1} t\right)$ and is therefore divisible by $R\left(a_{j}{ }^{(i)}\right)$, since the ideal of the inertia forms of these $n+1$ polynomials is, by the preceding theorems, 
a principal ideal and since the irreducible polynomial $R\left(a_{(j)}^{(i)}\right)$ obviously belongs to this ideal. It follows that $R\left(\cdots, t^{i_{1}+\cdots+j_{n}} a_{j_{1}}^{(i)} \cdots j_{n}, \cdots\right)$ differs from $R\left(a_{j}^{(i)}\right)$ only by a factor which is a power of $t$, say by $t^{\sigma}$. Hence $R\left(a_{j}^{(i)}\right)$ is an isobaric function of the coefficients of $a_{j}^{(i)}$, of weight $\sigma$, provided that we attach to $a_{j_{1}}^{(i)} \ldots j_{n}$ the weight $j_{1}+\cdots+j_{n}$.

To find $\sigma$, we specialize the polynomials $\phi_{i}$ as follows

$$
\phi_{1}=a_{1} x_{1}^{l_{1}}, \cdots, \phi_{n}=a_{n} x_{n}^{l_{n}}, \phi_{n+1}=a_{n+1} .
$$

The resultant $R$ does not vanish identically, since the equations $\phi_{1}=0, \cdots$, $\phi_{n+1}=0$ have no common solution if $a_{1}, \cdots, a_{n+1}$ are indeterminates. Taking into account the degree of $R$ in the coefficients of each $\phi_{i}$, we deduce that $R=c \cdot a_{1}^{l_{2}} \cdots l_{n+1} \ldots a_{n+1}^{l_{1} l_{2} \cdots l_{n}}$, where $c$ is a numerical factor. Since $a_{1}, \cdots, a_{n}$ are of weight $l_{1}, l_{2}, \cdots, l_{n}$ respectively and $a_{n+1}$ is of weight zero, it follows that $\sigma=n l_{1} l_{2} \cdots l_{n+1}$.

As an immediate corollary of this last result and of the fact that $R\left(\phi_{1}, \cdots, \phi_{n+1}\right)$ is homogeneous of degree $l_{1} \cdots l_{i-1} l_{i+1} \cdots l_{n+1}$ in the coefficients of $\phi_{i}$, it follows that if we attach to $a_{j_{1}}^{(i)} \cdots j_{n}$ the weight $l_{i}-j_{1}-\cdots-j_{n+1}$, then $R\left(\phi_{1}, \cdots, \phi_{n+1}\right)$ is isobaric of weight $l_{1} l_{2} \cdots l_{n+1}$.

7. Properties of $R$ based on a more general definition of the weights of the coefficients $a_{(j)}^{(i)}$. We separate in $\phi_{i}$ the terms of degree $\leqq s_{i}$ from those of degree $>s_{i}$, and we put $\phi_{i}=\bar{\psi}_{i}+\bar{f}_{i}$, where $\bar{\psi}_{i}$ is of degree $s_{i}$ and $\bar{f}_{i}$ contains all the terms of degree $>s_{i}$. While in $\$ 5$ we have replaced $a_{j_{1}}^{(i)} \ldots j_{n}$ by $t^{s_{i}-j_{1}-\cdots-j_{n}} a_{j_{1}}^{(i)} \ldots j_{n}$, if $s_{i}>j_{1}-\cdots-j_{n}$, we now instead replace $a_{j_{1}}^{(i)} \ldots j_{n}$ by $t^{j_{1}+\cdots+j_{n}-s_{1}} a_{j_{1}}^{(i)} \cdots j_{n}$, if $j_{1}+\cdots+j_{n}>s_{i}$, i.e., if $a_{j_{1}}^{(i)} \cdots j_{n}$ is the coefficient of a term in $\bar{f}_{i}$, and leave the coefficients of $\bar{\psi}_{1}, \cdots, \bar{\psi}_{n+1}$ unaltered.

Let $\bar{\phi}_{1}^{t}, \cdots, \bar{\phi}_{n+1}^{t}$ be the polynomials obtained in this manner, and let

$$
R\left(\bar{\phi}_{1}^{t}, \cdots, \bar{\phi}_{n+1}^{t}\right)=t^{\beta} R_{t}^{(2)}=t^{\beta} R^{(2)}\left(t ; a_{(j)}^{(i)}\right)
$$

be the resultant of the polynomials $\bar{\phi}_{i}{ }^{t}$. Here $t^{\beta}$ is the highest power of $t$ which divides $R\left(\bar{\phi}_{1}^{t}, \cdots, \bar{\phi}_{n+1}^{t}\right)$, so that $R_{0}^{(2)}=R^{(2)}\left(0 ; a_{j}^{(i)}\right)$ does not vanish identically. As in the case of the polynomials $\phi_{i}{ }^{t}$ of $\$ 5$, we conclude also here that $R_{0}^{(2)}$ is a form of inertia of the polynomials $\bar{\psi}_{1}, \cdots, \bar{\psi}_{n+1}$, and since these are general polynomials of degree $s_{1}, \ldots, s_{n+1}$ respectively, we deduce that $R_{0}^{(2)}$ is divisible by $R\left(\bar{\psi}_{1}, \cdots, \bar{\psi}_{n+1}\right)$.

Now the polynomials $\phi_{i}{ }^{t}$ and $\bar{\phi}_{i}{ }^{t}$ are related in the following way: $\bar{\phi}_{i}{ }^{t}=\phi_{i}{ }^{t}\left(t x_{1}, \cdots, t x_{n+1}\right) / t^{s_{i}}$. From this it follows, in view of the isobaric property of $R$ given in the preceding section, that their resultants differ only by a factor which is a power of $t$. Hence, by (7) and (8), we have $R^{(1)}\left(t, a_{(j)}^{(i)}\right)$ $=R^{(2)}\left(t, a_{(j)}^{(i)}\right)$, and in particular for $t=0$, we have $R_{0}^{(1)}=R_{0}^{(2)}$. Let $R_{0}=R_{0}^{(1)}$ $=R_{0}^{(2)} . R_{0}$ is divisible by $R\left(\bar{\psi}_{1}, \cdots, \bar{\psi}_{n+1}\right)$ and by $D$, where $D$ is the base of 
the principal ideal of the inertia forms of $f_{1}, \cdots, f_{n+1}$. $\dagger$ Hence $R_{0}$ is divisible by the product $D \times R\left(\bar{\psi}_{1}, \cdots, \bar{\psi}_{n+1}\right)$, since both factors are irreducible and distinct polynomials. $\left(R\left(\bar{\psi}_{1}, \cdots, \bar{\psi}_{n+1}\right)\right.$ is of degree $>0$ in each constant term $a_{00}^{(1)} \cdots_{0}$, while, except in the trivial case $s_{1}=\cdots=s_{n+1}=0$, where $f_{1}, \cdots, f_{n+1}$ coincide with $\phi_{1}, \cdots, \phi_{n+1}$, at least one of the polynomials $f$, say $f_{i}$, and hence also $D$, is independent of $a_{00}^{(1)} \ldots 0$.

The precise relationship between $R_{0}$ and $D \cdot R\left(\bar{\psi}_{1}, \cdots, \bar{\psi}_{n+1}\right)$ is given by the following theorems:

THEOREM 5.1. If two at least of the polynomials $f_{i}$ are non-homogeneous, then

$$
R_{0}=c \cdot D \cdot R\left(\bar{\psi}_{1}, \cdots, \bar{\psi}_{n+1}\right),
$$

where $c$ is a numerical factor (an integer).

THEOREM 5.2. If $f_{2}, \cdots, f_{n+1}$ are homogeneous, then

$$
R_{0}=c \cdot D^{l_{1-s_{1}}} \cdot R\left(\bar{\psi}_{1}, \cdots, \bar{\psi}_{n+1}\right),
$$

where $c$ is a numerical factor (an integer). In this case $D$ is simply the resultant of $f_{2}, \cdots, f_{n+1}$.

Before proving these theorems, let us first derive an immediate consequence. From the meaning of $R_{0}=R_{0}^{(1)}$ [cf. (7)] it follows that if to each coefficient $a_{j_{1}}^{(i)} \ldots i_{n}$ in $\phi_{i}$ we attach the weight $s_{i}-j_{1}-\cdots-j_{n}$, if $j_{1}+\cdots+j_{n}$ $\leqq s_{i}$, and the weight zero if $j_{1}+\cdots+j_{n}>s_{i}$, then $R_{0}$ is the sum of terms of owest weight $\alpha$ in the resultant $R\left(\phi_{1}, \cdots, \phi_{n+1}\right)$. According to this definition of the weight, each term in $D$ is of weight zero, while $R\left(\bar{\psi}_{1}, \cdots, \bar{\psi}_{n+1}\right)$, by $\S 6$, is of weight $s_{1} \cdots s_{n+1}$. Hence we may state the following theorem:

THEOREM 6. Let $\phi_{1}, \cdots, \phi_{n+1}$ be general polynomials in $x_{1} \cdots x_{n}$, of degree $l_{1}, \cdots, l_{n+1}$ respectively, and let $s_{1}, \cdots, s_{n+1}$ be integers such that $0 \leqq s_{i} \leqq l_{i}$. If we attach to each coefficient $a_{j_{1}}^{(i)} \cdots j_{n}$ in $\phi_{i}$ the weight $s_{i}-j_{1}-\cdots-j_{n}$ or the weight zero, according as $j_{1}+\cdots+j_{n} \leqq s_{i}$ or $j_{1}+\cdots+j_{n}>s_{i}$, then each term of the resultant $R\left(\phi_{1}, \cdots, \phi_{n+1}\right)$ is of weight $\geqq s_{1} s_{2} \cdots s_{n+1}$. The sum of terms of lowest weight $s_{1} s_{2} \cdots s_{n+1}$ is given by the product $c D^{\sigma} R\left(\bar{\psi}_{1}, \cdots, \bar{\psi}_{n+1}\right)$, where $c$ is a numerical factor. The symbols have the following meaning: $\bar{\psi}_{i}$ is the sum of terms of $\phi_{i}$ which are of degree $\leqq s_{i}$ and $f_{i}$ is the sum of terms of $\phi_{i}$ of degree $\geqq s_{i} ; R\left(\bar{\psi}_{1}, \cdots, \bar{\psi}_{n+1}\right)$ is the resultant of $\bar{\psi}_{1}, \cdots, \bar{\psi}_{n+1} ;$ if not all $s_{i}=l_{i}$, then $D$ is the base of the principal ideal of the inertia forms of $f_{1}, \cdots, f_{n+1}$; if all $s_{i}=l_{i}$, then $D=1$; finally, $\sigma=1$, except when all the integers $s_{i}$ but one, say $s_{1}$, coincide with the corresponding integers $l_{i}$, in which case $\sigma=l_{1}-s_{1}$.

$\dagger$ In the trivial case when $f_{1}, \cdots, f_{n+1}$ are all homogeneous polynomials, $D$ is not defined, but then $R_{0}$ evidently coincides with $R\left(\phi_{1}, \cdots, \phi_{n+1}\right)$. 
Remark. Again from the meaning of $R_{0}\left(=R_{0}{ }^{(2)}\right)$ it follows that if we attach to $a_{j_{1}}^{(i)} \cdots j_{n}$ the weight $j_{1}+\cdots+j_{n}-s_{i}$ or zero, according as $j_{1}+\cdots+j_{n} \geqq s_{i}$ or $j_{1}+\cdots+j_{n}<s_{i}$, then $R_{0}$ is also the sum of terms of lowest weight, $\beta$, in the resultant $R\left(\phi_{1}, \cdots, \phi_{n+1}\right)$ [cf. (8) ]. According to this definition of the weight, each term of $R\left(\bar{\psi}_{1}, \cdots, \bar{\psi}_{n+1}\right)$ is of weight zero, and $D^{\sigma}$ has to be isobaric of weight $\beta$.

To find $\beta$, we observe that Theorem 5.1 implies that $D$ is homogeneous of degree $l_{2} \cdots l_{n+1}-s_{2} \cdots s_{n+1}$ in the coefficients of $f_{1}$, homogeneous of degree $l_{1} l_{3} \cdots l_{n+1}-s_{1} s_{3} \cdots s_{n+1}$ in the coefficients of $f_{2}$, etc. On the other hand, if $j_{1}+\cdots+j_{n}$ is taken as the weight of $a_{j_{1}}^{(i)} \cdots j_{n}$, then $R_{0}$ and $R\left(\bar{\psi}_{1}, \cdots, \bar{\psi}_{n+1}\right)$ are isobaric forms of weight $n l_{1} \cdots l_{n+1}$ and $n s_{1} \cdots s_{n+1}$ respectively, whence $D$ is of weight $n\left(l_{1} \cdots l_{n+1}-s_{1} \cdots s_{n+1}\right)$. It follows that if we replace in the polynomial $D$ each coefficient $a_{j_{1}}^{(i)} \ldots j_{n}$ by $a_{j_{1}}^{(i)} \ldots j_{n} t^{s_{i}-j_{1}-\cdots-j_{n}}, D$ acquires the factor $t^{\beta}$, where

$$
\begin{aligned}
\beta= & -n\left(l_{1} \cdots l_{n+1}-s_{1} \cdots s_{n+1}\right)+s_{1}\left(l_{2} \cdots l_{n+1}-s_{2} \cdots s_{n+1}\right) \\
& +s_{2}\left(l_{1} l_{3} \cdots l_{n+1}-s_{1} s_{3} \cdots s_{n+1}\right)+\cdots+s_{n+1}\left(l_{1} \cdots l_{n}-s_{1} \cdots s_{n}\right),
\end{aligned}
$$

or

$$
\beta=s_{1} s_{2} \cdots s_{n+1}+\left(\frac{l_{1}-s_{1}}{l_{1}}+\cdots+\frac{l_{n+1}-s_{n+1}}{l_{n+1}}-1\right) l_{1} l_{2} \cdots l_{n+1} .
$$

If $s_{2}=l_{2}, \cdots, s_{n+1}=l_{n+1}$, then it is seen that $\beta=0$, and this agrees with Theorem 5.2 , because in this case the coefficients of $f_{2}, \cdots, f_{n+1}$ are of weight zero.

Proof of Theorems 5.1 and 5.2. We begin with Theorem 5.2, whose proof is simpler. We have in this case $\bar{\psi}_{i}=\phi_{i}, i=2, \cdots, n+1$, and hence $R\left(\bar{\psi}_{1}, \cdots, \bar{\psi}_{n+1}\right)$ is of degree $l_{2} \cdots l_{n+1}$ in the coefficients of $\bar{\psi}_{1}$. Hence, if we put

$$
R_{0}=D^{\sigma} R\left(\bar{\psi}_{1}, \phi_{2}, \cdots, \phi_{n+1}\right) \cdot P
$$

then $P$ is independent of the coefficients of $\phi_{1}$.

Now in the present case $\beta=0$, and $R_{0}$ is what becomes of the resultant $R\left(\bar{\phi}_{1}{ }^{t}, \cdots, \bar{\phi}_{n+1}^{t}\right)$ if we put $t=0$, where now $\phi_{i}{ }^{t}=\phi_{i}, i=2, \cdots, n+1$, and $\bar{\phi}_{1}{ }^{t}=\bar{\psi}_{1}+\bar{f}_{1}\left(t x_{1}, \cdots, t x_{n}\right) / t^{s_{1}}$. It follows that $R_{0}=0$ implies that either the equations $\bar{\psi}_{1}=0, \phi_{2}=0, \cdots, \phi_{n+1}=0$, rendered homogeneous, have a nontrivial solution, or that the homogeneous equations $f_{2}=0, \cdots, f_{n+1}=0$ have a non-trivial solution. Hence $R\left(\bar{\psi}_{1}, \phi_{2}, \cdots, \phi_{n+1}\right)$ and $R\left(f_{2}, \cdots, f_{n+1}\right)$ are the only irreducible factors which can occur in $R_{0}$. Since the irreducible poly- 
nomial $R\left(f_{2}, \cdots, f_{n+1}\right)$ obviously coincides with $D$, Theorem 5.2 follows by comparing the degrees of the first and second member of (9.2).

For the proof of Theorem 5.1, it is sufficient to show that $D$ is of degree $l_{2} l_{3} \cdots l_{n+1}-s_{2} s_{3} \cdots s_{n+1}$ in the coefficients of $f_{1}$, of degree $l_{1} l_{3} \cdots l_{n+1}-s_{1} s_{3} \cdots$ $s_{n+1}$ in the coefficients of $f_{2}$, etc. Since $D R\left(\bar{\psi}_{1}, \cdots, \bar{\psi}_{n+1}\right)$ divides $R_{0}, D$ cannot be of higher degree in the coefficients of $f_{1}, f_{2}, \cdots, f_{n+1}$, and therefore it remains to show that $D$ is of degree not less than $l_{2} l_{3} \cdots l_{n+1}-s_{2} s_{3} \cdots s_{n+1}$ in the coefficients of $f_{1}$, etc. We prove this in the following section.

8. The degree of $D$. We wish to show in this section that if at least two of the polynomials $f_{1}, \cdots, f_{n+1}$ are non-homogeneous, then $D$ is of degree $\geqq l_{2} \cdots l_{n+1}-s_{2} \cdots s_{n+1}$ in the coefficients of $f_{1}$, of degree $\geqq l_{1} l_{3} \cdots l_{n+1}-s_{1} s_{3}$ $\cdots s_{n+1}$ in the coefficients of $f_{2}$, etc. Obviously, the condition that at least two of the polynomials $f_{i}$ be non-homogeneous, is necessary. In fact, if only one of the polynomials $f_{i}$, say $f_{n+1}$, is non-homogeneous, then $D$ coincides with the resultant $R\left(f_{1}, \cdots, f_{n}\right)$ of the forms $f_{1}, \cdots, f_{n}$, and its degree in the coefficients of $f_{1}$ is not $l_{2} \cdots l_{n+1}-s_{2} \cdots s_{n+1}\left[=l_{2} \cdots l_{n}\left(l_{n+1}-s_{n+1}\right)\right]$, but $l_{2} \cdots l_{n}$. If all the polynomials $f_{i}$ are homogeneous, then the ideal of their inertia forms is not a principal ideal and $D$ is not defined.

If for special values of the coefficients $a_{(j)}^{(i)}$, one of the polynomials $f_{i}$, say $f_{n+1}$, factors into a product $g h$ of two polynomials, then $D$ becomes an inertia form of both sets of polynomials $f_{1}, \cdots, f_{n}, g$ and $f_{1}, \cdots, f_{n}, h$. Hence, assuming that the ideals of inertia forms of these two sets of polynomials are principal ideals, say $\left(D_{1}\right)$ and $\left(D_{2}\right)$ respectively, then for those special values of the coefficients $a_{(j)}^{(i)}, D$ is divisible by both $D_{1}$ and $D_{2}$. This remark shall be used in the sequel.

Let $f_{n}$ and $f_{n+1}$ be the non-homogeneous polynomials. We first consider the case in which $f_{1}, \cdots, f_{n-1}$ are polynomials of degree 1 , and in this case we examine separately three possibilities.

(a) At least two of the polynomials $f_{1}, \cdots, f_{n-1}$ are non-homogeneous (and hence two at least of the integers $s_{1}, \cdots, s_{n-1}$ vanish). We specialize the coefficients of $f_{n}$ and $f_{n+1}$ in such a manner that $f_{n}$ becomes the product of $l_{n}$ general polynomials $f_{n, i}$ of the first degree, of which $s_{n}$ are linear forms, and that $f_{n+1}$ becomes similarly the product of $l_{n+1}$ linear factors, $f_{n+1, i}$. The $l_{n} l_{n+1}$ $(n+1)$-row coefficient determinants relative to the sets of polynomials $f_{1}, \cdots, f_{n-1}, f_{n, i}, f_{n+1, j}$ are all distinct and irreducible inertia forms, since at least two of the polynomials of each set are non-homogeneous. Hence $D$ is divisible by the product of these determinants and is therefore of degree $\geqq l_{n} l_{n+1}$ in the coefficients of $f_{i}, i=1,2, \cdots, n-1$, and of degree $\geqq l_{n+1}\left(l_{n}\right)$ in the coefficients of $f_{n}\left(f_{n+1}\right)$.

We observe that this proves that in the present case $D$ coincides with the 
resultant $R\left(f_{1}, \cdots, f_{n+1}\right)$, or, what is the same, that this resultant is irreducible.

(b) All but one of the polynomials $f_{1}, \cdots, f_{n-1}$ are homogeneous. Let, for instance, $f_{1}$ be non-homogeneous. With the same specialization of $f_{n}$ and $f_{n+1}$ as in the preceding case, let $f_{n, 1}, \cdots, f_{n, s_{n}} ; f_{n+1,1}, \cdots, f_{n+1, s_{n+1}}$ be the homogeneous linear factors of $f_{n}$ and of $f_{n+1}$ respectively. The $(n+1)$-row coefficient determinants of $f_{1}, \cdots, f_{n-1}, f_{n, i}, f_{n+1, j}$ remain irreducible, except when simultaneously $1 \leqq i \leqq s_{n}$ and $1 \leqq j \leqq s_{n+1}$, in which case the determinant factors into the constant term of $f_{1}$ and into the $n$-row determinant of the coefficients of $x_{1}, \cdots, x_{n}$ in $f_{2}, \cdots, f_{n-1}, f_{n, i}, f_{n+1, j}$. Hence $D$ is divisible by the product of $l_{n} l_{n+1}-s_{n} s_{n+1}(n+1)$-row determinants and $s_{n} s_{n+1} n$-row determinants, these last ones being independent of the coefficients of $f_{1}$. Hence $D$ is of degree $\geqq l_{n} l_{n+1}-s_{n} s_{n+1}$ in the coefficients of $f_{1}$, of degree $\geqq l_{n} l_{n+1}$ in the coefficients of $f_{i}, i=2, \cdots, n-1$, and of degree $\geqq l_{n+1}\left(l_{n}\right)$ in the coefficients of $f_{n}\left(f_{n+1}\right)$.

(c) All the polynomials $f_{1}, \cdots, f_{n-1}$ are homogeneous. Let

$$
\begin{aligned}
f_{i} & =\sum_{j=1}^{n} a_{i j} x_{i}, \quad i=1,2, \cdots, n-1, \\
f_{i} & =f_{i, s_{i}}+f_{i, s_{i}+1}+\cdots+f_{i, l_{i}}, \quad i=n, n+1,
\end{aligned}
$$

where $f_{i, s_{i}+k}$ is homogeneous of degree $s_{i}+k$. Solving (10) for $x_{2}, \cdots, x_{n}$ we get

$$
A_{1} x_{i} \equiv A_{i} x_{1}\left(f_{1}, f_{2}, \cdots, f_{n-1}\right),
$$

where $A_{1}, \cdots, A_{n}$ are $(n-1)$-row minors of the matrix $\left(a_{i j}\right)$ and hence homogeneous of degree 1 in the coefficients of each of the polynomials $f_{1}, \cdots, f_{n-1}$.

Substituting (11) into $\left(10^{\prime}\right)$ we get

$$
A_{1}^{l_{n}} f_{n} \equiv x_{1}^{s_{n}} \phi_{n}\left(x_{1}\right)\left(f_{1}, \cdots, f_{n-1}\right) ; A_{1}^{l_{n+1}} f_{n+1} \equiv x_{1}^{s_{n+1}} \phi_{n+1}\left(x_{1}\right)\left(f_{1}, \cdots, f_{n-1}\right),
$$

where

$$
\begin{aligned}
\phi_{n}\left(x_{1}\right)= & A_{1}^{l_{n-8 n}} f_{n, s_{n}}\left(A_{1}, \cdots, A_{n}\right)+x_{1} A_{1}^{l_{n-s_{n}-1}} f_{n, s_{n}+1}\left(A_{1}, \cdots, A_{n}\right)+\cdots \\
& +x_{1}^{l_{n}-s_{n}} f_{n, l_{n}}\left(A_{1}, \cdots, A_{n}\right) \\
\phi_{n+1}\left(x_{1}\right)= & A_{1}^{l_{n+1-8 n+1}} f_{n+1, s_{n+1}}\left(A_{1}, \cdots, A_{n}\right) \\
& +x_{1} A_{1}^{l_{n+1-s_{n+1}-1}} f_{n+1, e_{n+1}+1}\left(A_{1}, \cdots, A_{n}\right)+\cdots \\
& +x_{1}^{l_{n+1-s_{n+1}}} f_{n+1, l_{n+1}}\left(A_{1}, \cdots, A_{n}\right) .
\end{aligned}
$$


Let $R=R\left(\phi_{n}, \phi_{n+1}\right)$ be the resultant of $\phi_{n}\left(x_{1}\right)$ and $\phi_{n+1}\left(x_{1}\right)$. We have $R \equiv 0\left(\phi_{n}, \phi_{n+1}\right)$ and hence, by (12), $x_{1}{ }^{\sigma} R \equiv 0\left(f_{1}, \cdots, f_{n-1}, f_{n}, f_{n+1}\right)$, for some $\sigma$. It follows, by Theorem 2 , that $R$ is a form of inertia of the polynomials $f_{i}$. From the form of the coefficients of $\phi_{n}\left(x_{1}\right)$ and $\phi_{n+1}\left(x_{1}\right)$ and from the fact that $R$ is an isobaric form of weight $\left(l_{n}-s_{n}\right)\left(l_{n+1}-s_{n+1}\right)$ in these coefficients, it follows that $A_{1}{ }^{\left(l_{n}-s_{n}\right)\left(l_{n+1}-s_{n+1}\right)}$ is a factor of $R$. Let $R=A_{1}{ }^{\left(l_{n-s_{n}}\right)\left(l_{n+1}-s_{n+1}\right)} \cdot P$. Now $A_{1}$ is independent of the coefficients of $f_{n}$ and $f_{n+1}$ and hence, by Theorem 4 , is not a form of inertia of $f_{1}, \cdots, f_{n+1}$. Consequently $P$ is a form of inertia of $f_{1}, \cdots, f_{n+1}$. The coefficients of $\phi_{i}(i=n, n+1)$ are homogeneous of degree 1 in the coefficients of $f_{i}$ and homogeneous of degree $l_{i}$ in $A_{1}, \cdots, A_{n}$, hence homogeneous of degree $l_{i}$ in the coefficients of each of the polynomials $f_{1}, \cdots, f_{n-1}$. Hence $R$ is homogeneous of degree $l_{n}-s_{n}$ and $l_{n+1}-s_{n+1}$ in the coefficients of $f_{n+1}$ and $f_{n}$ respectively, and homogeneous of degree $l_{n}\left(l_{n+1}-s_{n+1}\right)+l_{n+1}\left(l_{n}-s_{n}\right)$ in the coefficients of $f_{i}, i=1,2, \cdots, n-1$. It follows that $P$ is homogeneous of degree $l_{n+1}-s_{n+1}$ and $l_{n}-s_{n}$ in the coefficients of $f_{n}$ and $f_{n+1}$ respectively, and homogeneous of degree $l_{n} l_{n+1}-s_{n} s_{n+1}$ in the coefficients of each of the polynomials $f_{1}, \cdots, f_{n-1}$.

It remains to prove that $P=D$, or, what is the same, that $P$ is an irreducible polynomial in the coefficients of $f_{1}, \cdots, f_{n+1}$. We observe that $P$ is the resultant of the following polynomials

$$
\begin{aligned}
\psi_{n}\left(x_{1} ; A_{1}, \cdots, A_{n}\right)= & f_{n, s_{n}}\left(A_{1}, \cdots, A_{n}\right)+x_{1} f_{n, s_{n+1}}\left(A_{1}, \cdots, A_{n}\right) \\
& +\cdots+x_{1}^{l_{n-s_{n}}} f_{n, l_{n}}\left(A_{1}, \cdots, A_{n}\right), \\
\psi_{n+1}\left(x_{1} ; A_{1}, \cdots, A_{n}\right)= & f_{n+1, s_{n+1}}\left(A_{1}, \cdots, A_{n}\right)+x_{1} f_{n+1, s_{n+1}+1}\left(A_{1}, \cdots, A_{n}\right) \\
& +\cdots+x_{1}^{l_{n+1-s_{n+1}}} f_{n+1, l_{n+1}}\left(A_{1}, \cdots, A_{n}\right) .
\end{aligned}
$$

For the special polynomials $f_{1}=x_{2}, f_{2}=x_{3}, \cdots, f_{n-1}=x_{n}$, we have $A_{1}=1$, $A_{2}=\cdots=A_{n}=0$, and $\psi_{n}, \psi_{n+1}$ become general polynomials with literal coefficients in $x_{1}$, of degree $l_{n}-s_{n}$ and $l_{n+1}-s_{n+1}$ respectively, and their resultant is irreducible. Hence $P$ cannot be divisible by two factors or by the square of a factor in which the coefficients of $f_{n}$ or of $f_{n+1}$ actually occur. On the other hand, for the special polynomials

$$
\begin{aligned}
\psi_{n} & =x_{1}^{l_{n-8 n}} f_{n, l_{n}}\left(A_{1}, \cdots, A_{n}\right), \\
\psi_{n+1} & =f_{n+1,8 n+1}\left(A_{1}, \cdots, A_{n}\right)+x_{1}^{l_{n+1-8 n+1}} f_{n+1, l_{n+1}}\left(A_{1}, \cdots, A_{n}\right)
\end{aligned}
$$

we get $P= \pm f_{n, l_{n}}^{l_{n+1}-s_{n+1}} f_{n+1}^{h_{n}, s_{n}}, l_{n+1}$, and hence $P$ cannot have a factor independent of the coefficients of both $f_{n}$ and $f_{n+1}$. Hence $P$ is irreducible, $P=D$.

Passing to the general case where $f_{1}, \cdots, f_{n-1}$ are of arbitrary degrees $l_{1}, \cdots, l_{n-1}$, while $f_{n}, f_{n+1}$ are non-homogeneous polynomials, we specialize 
each polynomial $f_{i}, i=1,2, \cdots, n-1$, into the product of $l_{i}$ linear factors, of which $s_{i}$ are linear forms: $f_{i}=f_{i 1} f_{i 2} \cdots f_{i l_{i}}$. By the special case considered above, the irreducible form of inertia $D_{j_{1} \ldots j_{n-1}}$ of the $n+1$ polynomials $f_{1 j_{1}}, f_{2 j_{2}}, \cdots, f_{n-1, j_{n-1}}, f_{n}, f_{n+1}\left(1 \leqq j_{i} \leqq l_{i}\right)$ actually contains the coefficients of each factor. Hence we get $l_{1} l_{2} \ldots l_{n-1}$ distinct irreducible forms of inertia and their product must divide $D$. Now $D_{j_{1}} \ldots j_{n-1}$ is of degree $l_{n} l_{n+1}$ in the coefficients of $f_{1 j_{1}}$, if the polynomials $f_{2 j_{2}}, \cdots, f_{n-1, j_{n-1}}$ are not all homogeneous, and is of degree $l_{n} l_{n+1}-s_{n} s_{n+1}$ in the coefficients of $f_{1 j_{1}}$, if all the polynomials $f_{2 j_{2}}, \cdots, f_{n-1, j_{n-1}}$ are homogeneous. It follows that $D$ is of degree $\geqq l_{2} \cdots l_{n+1}-s_{2} \cdots s_{n+1}$ in the coefficients of $f_{1}$. Similarly $D$ is of degree $\geqq l_{1} \cdots l_{i-1} l_{i+1} \cdots l_{n+1}-s_{1} \cdots s_{i-1} s_{i+1} \cdots s_{n+1}$ in the coefficients of $f_{i}$, $i=1,2, \cdots, n-1 . D_{i_{1}} \cdots j_{n-1}$ is of degree $l_{n+1}$ in the coefficients of $f_{n}$, if $f_{1 i_{1}}, \cdots, f_{n-1, j_{n-1}}$ are not all homogeneous, and is of degree $l_{n+1}-s_{n+1}$ in the contrary case. Hence $D$ is of degree $l_{1} \cdots l_{n-1} l_{n+1}-s_{1} \cdots s_{n-1} s_{n+1}$ in the coefficients of $f_{n}$. Similarly, $D$ is of degree $l_{1} \cdots l_{n}-s_{1} \cdots s_{n}$ in the coefficients of $f_{n+1}$.

9. An application to the intersection theory of algebraic hypersurfaces. Let

$$
\begin{gathered}
\phi_{1}\left(x_{1}, \cdots, x_{n}, x_{n+1}\right)=0 \\
\cdots \cdots \\
\phi_{n+1}\left(x_{1}, \cdots, x_{n}, x_{n+1}\right)=0
\end{gathered}
$$

be the equations of $n+1$ hypersurfaces $F_{1}, \cdots, F_{n+1}$ in the $(n+1)$-dimensional projective space. Let $l_{i}$ be the order of $F_{i}$. Let the origin $O(0, \cdots, 0)$ be a common point of these hypersurfaces, and let it be an $s_{i}$-fold point of $F_{i}$. We regard $\phi_{i}$ as a polynomial in $x_{1}, \cdots, x_{n}$, and we write $\phi_{i}=\sum a_{j_{1}}^{(i)} \ldots j_{n} x_{1} i_{1} \ldots x_{n}^{j_{n}}$, where the coefficients $a_{(j)}^{(i)}$ are polynomials in $x_{n+1}$. Since $O$ is an $s_{i}$-fold point of $F_{i}, a_{j_{1}}^{(i)} \ldots j_{n}$ is divisible by $x_{1}^{s_{i}-j_{1}-\cdots-j_{n}}$, if $j_{1}+\cdots+j_{n} \leqq s_{i}$. Hence, by Theorem 6 , every term of the resultant $R\left(\phi_{1}, \cdots, \phi_{n+1}\right)=R\left(x_{n+1}\right)$ is divisible by $x_{n+1}^{s_{1} \cdots s_{n+1}}$. Let

$$
R\left(x_{n+1}\right)=\alpha x^{s_{1} \cdots s_{n+1}}+\text { terms of higher degree, }
$$

where $\alpha$ is a constant. Let $g_{i}(i=1,2, \cdots, n+1)$ denote the sum of terms of lowest degree $\left(s_{i}\right)$ in $\phi_{i}$. Then we have, by Theorem $6, \alpha=c D_{0}{ }^{\sigma} R\left(g_{1}, \cdots, g_{n+1}\right)$, where $c$ is an integer, and $D_{0}=[D]_{x_{n+1}=0}$. The homogeneous equation $g_{i}=0$ represents the tangent hypercone of the hypersurface $F_{i}$ at the point $O$. Hence $R\left(g_{1}, \cdots, g_{n+1}\right)$ vanishes, if and only if the $n+1$ hypersurfaces $F_{i}$ have a common principal tangent line at $O$. Assume that $R\left(g_{1}, \cdots, g_{n+1}\right) \neq 0$. If $f_{i}$ 
denotes, as in Theorem 6 , the sum of terms in $\phi_{i}$ which are of degree $\geqq s_{i}$ in $x_{1}, \cdots, x_{n}$, then $f_{i}=\left[g_{i}\right]_{x_{n+1}=0}+$ terms of degree $>s_{i}$ in $x_{1}, \cdots, x_{n}$. It follows, by Theorem 3.1, that if $R\left(g_{1}, \cdots, g_{n+1}\right) \neq 0$, then $D_{0}=0$ implies that the hypersurfaces $F_{i}$ have a common point on the hyperplane $x_{n+1}=0$, outside the origin; and conversely, by Theorem 3.2. Assuming that the hypersurfaces $F_{i}$ meet in a finite number of points, we see that if the coordinate axes are in generic position and if the hypersurfaces $F_{i}$ have no principal tangent in common at the point $O$, then $\alpha \neq 0$. According to the usual definition of the intersection multiplicity of the hypersurfaces $F_{i}$ at a common point, it follows that the intersection multiplicity at $O$ is $\geqq s_{1} \cdots s_{n+1}$ and equals $s_{1} \cdots s_{n+1}$ if and only if the hypersurfaces $F_{i}$ have no common principal tangent at $O$.

The Johns Hopkins University, BALTIMORE, MD. 